\title{
The Design and Implementation of Random Soft Keyboard Based on Sin- gle Chip
}

\author{
Song Tian", Tao Liu and Jinyu Wei \\ Department of Computer, Hubei Polytechnic University, Huangshi, Hubei, 435000, China
}

\begin{abstract}
There are a great amount of potential security threats during e-commerce, banking business and securities trading by fixed password input devices, drawing more and more attention. In order to prevent passwords from illegally snooping or stealing, a lot of methods are needed to stop fund loss etc. With the purpose of resolving the issue, the author of the design comes up with a plan that uses software to produce dynamic and random layout of keyboard, reducing the probability of stolen passwords to a large extent. The development tools of the design are PROTEUS and KEIL-C. Under PROTEUS environment, the keyboard layout, operation instructions of function modules, selection and layout of components, as well as man-machine interaction are constructed. Under KEIL-C environment, code for the randomly generated module, code for the right button module, code for the random keyboard display module, source code for the password display module, time delay module, module for password input and process, module for password modification and main program module are completed. Each module is displayed smoothly, and with the help of Single chip, the system could be with high feasibility and low development costs, meaningful for the practical application field.
\end{abstract}

Keywords: Random keyboard, PROTEUS, Simulation interface, Man-machine interaction.

\section{INTRODUCTION}

With the input of transaction number in e-commerce, bank ATM and securities trading, physical keyboard and electronic keyboard are more and more popular, so personal and collective information security of users, safety of passwords, in particular, play a significant role as the society develops. The issues that some common password input devices are in danger of leaking passwords for using too many times draws more and more attention. Generally, the solution is to change passwords or password input devices [1-3]. These solutions, however, leave a lot of problems. For instance, frequently changing passwords can be a major inconvenience to the users, while changing password input devices can still leave some potential password security threats. In order to prevent users' passwords being stolen or used by fraudulent people, the author puts forward a solution that applies simulation software to create dynamic layout of keyboard, with the password display order in random when the buttons are used with same probability, making fraudulent people unable to steal the secret according to their usual experiences [4], thus significantly eliminate the password leaking threat and reduce the probability of stolen passwords.

\section{OVERALL DESIGNS}

\subsection{Overall Requirements}

The design needs to make relevant schematic diagram by PROTEUS simulation software. The schematic diagram should include password keyboard display module, keyboard input module, password setting module, right password

*Address correspondence to this author at the Department of Computer, Hubei Polytechnic University, Huangshi, Hubei, 435000, China; Tel: 13092771669; E-mail: 12093498@qq.com display module and wrong password display module. It requires to conduct program and code development under KEIL-C environment, designing codes for each module, namely module code, code for the right button module, code for the random keyboard display module, source code for the password display module, time delay module, module for password input and process, module for password modification and main program module etc. the program should be written into. HEX program and conducted simulation debugging and development. Finally, it should be smoothly connected and shows the function of random dynamic keyboard coded lock.

\subsection{Development Environment}

\subsubsection{Proteus}

Proteus is developed by Labcenter Electronics, an England based company. Besides the same schematic diagram layout, PCB automatic or manual wiring as well as circuit simulation like other EDA tools, it features simulation interactive circuits, is able to directly program in the virtual prototype of schematic diagram and realizes real-time software debugging in source code level regarding the application of microprocessor, such as viewing the effect of input and output of operation when displaying, outing and simulating. Therefore, Proteus establishes a complete electronic development and design environment for the developers, convenient for interaction with developers [5, 6], Proteus is shown as Fig. (1).

\section{DETAILED DESIGN}

\subsection{Design and Layout of Schematic Diagram Under PROTEUS Environment}

According to the design thought of random dynamic coded lock, it requires to design schematic diagram under PRO- 


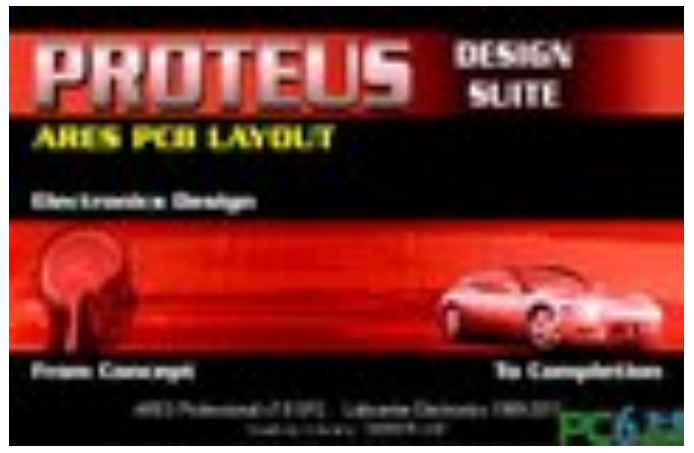

Fig. (1). Proteus software.

TEUS environment. The simulation schematic diagram should include display module, password inputting module, components connection and layout as well as the alert when inputting passwords. The components are connected as Fig. (2):

\subsubsection{Keil uVision3}

MCS-51compiler from Keil Company, one of the excellent softwares in Single chip, application software, supports chips of MCS-51 structure of many companies, integrating edition, compiling and simulation in one. It also supports PLM, assembly and C language program design [7, 8], with friendly interface similar to Microsoft VC interface, with strong debugging and software simulation functions, being a common Single chip, C language compiler. UVision3 launched by Keil Software Company provides collective development environment that could be applied in all sorts of 51 Single chip, compiler. Thus the design should also adopt such software for development $[9,10]$.

\subsection{Division of Major Modules}

1) Design of Proteus schematic diagram: layout by selecting relevant components.

2) Beautify schematic diagram: according to the design requirement.

3) Display the number corresponding to the random soft keyboard: displaying different content by pressing different numbers.

4) Function of inputting passwords: with different signals when inputting right or wrong passwords.

5) Invisible indication when inputting passwords: not showing passwords when inputting.

6) Indication of changing passwords: when changing new passwords, it shall be inputted twice.

\subsection{Detailed Design of Each Submodule}

\subsubsection{Code for the Randomly Generated Module}

The module generates algorithm of keyboard, making numbers in keyboard in disorder. Place the numbers in keyboard in array, and exchange the former five numbers with latter five numbers, then exchange them across one number, and exchange them across two number and then across three number. Finally the former five numbers are exchanged with latter five numbers. The disorder of keyboard is achieved by operating the array.

\subsubsection{Code for the Right Button Module}

Input data and turn back. The display of keyboard is realized by an array. When pressing buttons, inquire the pressed button and check the array to make sure the corresponding

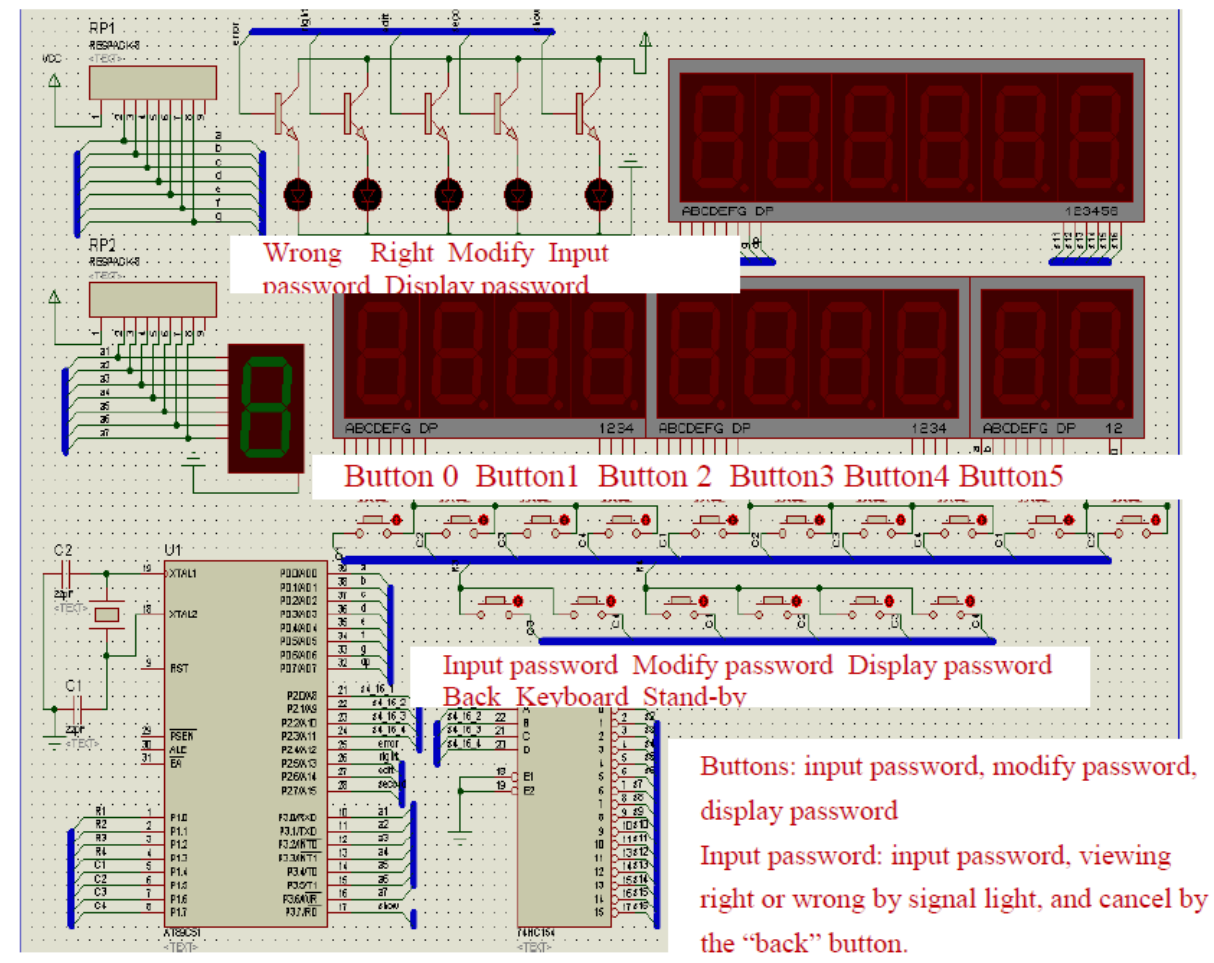

Fig. (2). Simulation design diagram. 
number. The innovation of the design is holding and pressing a button does not input information. Pressing more than one button will only reserve the information of the first pressing.

\subsubsection{Code for the Random Keyboard Display Module, Source code for the Password Display Module}

Realize the display of random keyboard by using interrupting 0 in type 1 of 51 Single chip,.

\subsubsection{Time Delay Module}

The clock of Single chip, is in 12MHZ. During operation, the low manual speed is unmatched with mechanical high speed so it needs delay time. Besides, the Single chip, have to delay time because it takes time for the outside components of Single chip, to transport.

\subsubsection{Module for Password Input and Process}

The module inputs password and judges whether it is right or not. It realizes password modification, by inputting original password (if wrong, re-input or turn back), and inputs new password for twice [11]. if the two new passwords are not the same, new password should be inputted twice again or turn back, otherwise, the password is modified successfully.

\subsubsection{Main Programs}

Main programs include: relevant data of buttons in keyboard, making sure the displaying way of password (display or does not display), changing keyboard, selection of input password function, selection of password modification.

\subsubsection{Selection and Layout of Components}

Selecting components:

AT89C51,7SEG-COM-CAT-GRN,7SEG-MPX2-CC,7SEG-MPX4-CC,7SEG-MPX6-CC,74HC154, BUTTON, CAP, CARYSTAL,LED-BIBY,LED-BIGY,LED-BIRG, LED-BIRY, LED-BLUE,LED-GREEN,LED-RED,LED-YELLOW， NPN, RES,RESPACK-8. Wire the components according to the schematic diagram after placing them well. Wiring should be clear without overlapping, like shown in Fig. (2).

\section{DESIGN TEST PROGRAM}

\subsection{Reasonable Design of Schematic Diagram}

Under KEIL-C environment, test the designed schematic diagram. Select testing tools of schematic diagram under TOOL menu. A successful test is shown as Fig. (3).

\subsection{Code Operation Under KEIL-C Environment}

\subsubsection{Proteus}

Proteus is developed by Labcenter Electronics, an England based company. Besides the same schematic diagram layout, PCB needs automatic or manual wiring as well as circuit simulation like other EDA tools. According to the requirements of environment, under KEIL-C environment, the model of Single chip is AT89C51 series: the designed random soft keyboard creates code for the randomly generated module, code for the right button module, code for the random keyboard display module, source code for the password display module, time delay module, module for pass- word input and process, and module for password modification.

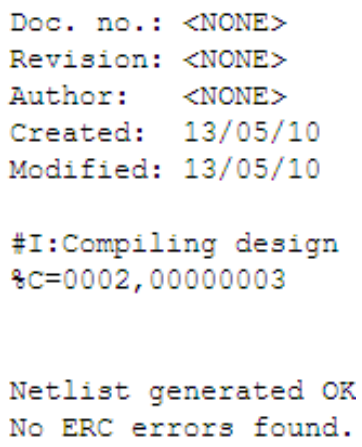

Fig. (3). The result of right placement of component.

The design should be saved in two forms: ASM and C. The design is operated under .C environment: firstly establish "NEW PROJECT" under PROJECT and write the program module according to requirement, then save it as .C program. Load the program in the project, select "ADD FILES TO GROUP SOURCE GROUP 1", under FILE menu, and select OUTPUT .HEX in the dialog, and then operate the program and keep debugging and modifying it until it satisfies the requirement of the design by appearing "0ERRORS 0WARNINGS". Then generate .HEX program and save it in a file. Open the designed DSN file, and click the AT89C51 chip. Then add the written .HEX file to the chip and operate. It shows code for the randomly generated module, code for the right button module, source code for the password display module, module for password display etc. under KEIL-C environment, smoothly connecting to display, modify, re-set the random passwords.

\section{CONCLUSION}

The system is random password keyboard technology developed under simulation environment, and is meaningful for the practical application in information safety field.

The development tools of the design are PROTEUS and KEIL-C. Under PROTEUS environment, the keyboard layout, operation instructions of function modules, selection and layout of components, as well as man-machine interaction are constructed. Under KEIL-C environment, code for the randomly generated module, code for the right button module, code for the random keyboard display module, source code for the password display module, time delay module, module for password input and process, module for password modification and main program module are completed. Each module is displayed smoothly, and the interface has elegant appearance and is meaningful for the practical application field.

There are several achievements of the design:

1) Used as development tool, with advantages of clear tough design, complete components and clear LCD digital display.

2) Write code with $C$ language under KEIL-C environment, with advantages of convenient design of program, convenience for error correction and portability. It can finish function modules needed constantly and realize them. 
3) Low power consumption. Designing random dynamic coded lock by single chip is with low power consumption, low cost and good exploitation, meaningful for mass production.

4) Put forward a new tough pattern based on Single chip,, gradually replacing development of original soft keyboard and is widely applied in each field.

\section{CONFLICT OF INTEREST}

The authors confirm that this article content has no conflict of interest.

\section{ACKNOWLEDGEMENTS}

This work was supported by the No.1 project "Open Laboratory Fund Project of Hubei Polytechnic University (project No.: 31), Research on integrated design of innovative experiment based on single chip microcomputer". No.2 project" Undergraduate innovative experiment of Hubei Polytechnic University (project No.: 5), Design and implementation of voice system based on SPCE061", The authors wish to thank the Hubei Polytechnic University for their help.

\section{REFERENCES}

[1] C. Liu, "Snooping-proof Password Keyboard with Rolling Digital Password Concept," Electronic Technology Applications, pp.19-20, 2004.

[2] W. Xu, "Proteus Software Application in simulation Experiment Teaching of Singlechip System," Business Economy, no. 3, pp. 9093, 2006.

[3] F. Wang, et al, "Keil and Proteus Application in Modern Teaching," Modern Enterprise Education, no. 6, pp. 78-80, 2009.

[4] Z. Liu, "Exploration of Key Technology and Mode of Business of Internet of Things," Guangdong Communication Technology, no. 12, 2009.

[5] H. Hu, "Principle and Design of Singlechip," Version 2, Tsinghua University Press, 2004.

[6] R. Zhou, and L. Zhang, "Design and Simulation of Circuits and Singelchip System based-on PROTEUS," Version 2, Beijing University of Aeronautics and Astronautics Press, 2006.

[7] H. Tan, "Design of C Language Program," Version 1, Tsinghua University Press, 2010.

[8] J. Zhang, et al, "Dynamic Plan of Shortest Path and C Language Realization," Journal of Inner Mongolia Agricultural University (Natural Sciences), vol. 33, no.2, 2012.

[9] X. Wu, "Application of C Language in Singlechip Technology," vol. 29 , no. 36 , pp. 2010.

[10] S. Cao, "Research and Thought of C Language Teaching," Value Engineering, vol. 31, no.1, 2012.

[11] T. Wang, "Discuss of Teaching Method of C Language," Computer CD Software and Applications, no.9, 2011.

(C) Tian et al.; Licensee Bentham Open.

This is an open access articles licensed under the terms of the Creative Commons Attribution-Non-Commercial 4.0 International Public License (CC BY-NC 4.0) (https://creativecommons.org/licenses/by-nc/4.0/legalcode), which permits unrestricted, non-commercial use, distribution and reproduction in any medium, provided that the work is properly cited. 